\title{
TRANSVERSE EFFECT DUE TO SHORT-RANGE RESISTIVE WALL WAKEFIELD*
}

\author{
Juhao $\mathrm{Wu}^{\dagger}$, Alexander W. Chao, Stanford Linear Accelerator Center, Menlo Park, CA 94025, USA, \\ Jean R. Delayen, Thomas Jefferson National Accelerator Facility, Newport News, Virginia, 23606, \\ Old Dominion University, Norfolk, Virginia, 23529, USA
}

\section{Abstract}

For accelerator designs with ultra short electron beams, beam dynamics study has to invoke the short-range wakefields. In this paper, we first obtain the short-range dipole mode resistive wall wakefield. Analytical approach is then developed to study the single bunch transverse beam dynamics due to this short-range resistive wall wake. The results are applied to the LCLS undulator.

\section{Introduction}

If the source of the wakefield is the resistive wall of a circularly cylindrical beam pipe, then the long-range dipole wakefield is $[1,2] \mathcal{W}(\zeta)=\varepsilon_{l} / \sqrt{\zeta}$ for $\zeta>0$, where $\zeta=t-s / v$ is the relative longitudinal position of the particle from the front of the beam; $v$ is the particle velocity; $\varepsilon_{l}=\left(4 c^{2} \bar{I}\right) /\left(v \gamma b^{3} I_{\mathrm{A}}\right) \sqrt{\epsilon_{0} /\left(\pi \sigma_{c}\right)}$ with $c$ being the speed of light in vacuum, $\gamma$ the Lorentz factor, $b$ the pipe radius, $I_{\mathrm{A}}=4 \pi \epsilon_{0} m c^{3} / e \approx 17,045 \mathrm{Amp}$ the Alfvèn current, $\epsilon_{0}=8.8542 \times 10^{-12} \mathrm{C}^{2} /\left(\mathrm{N} \mathrm{m}^{2}\right)$ the vacuum permittivity, and $\sigma_{c}$ the pipe conductivity. Note that $\mathcal{W}(\zeta)=\varepsilon_{l} / \sqrt{\zeta}$ is valid only for $[2,3]$

$$
\left(\frac{b^{2}}{Z_{0} \sigma_{c} c^{3}}\right)^{1 / 3} \ll \zeta \ll \min \left[\frac{Z_{0} \sigma_{c} b^{2}}{c}, \frac{Z_{0} \sigma_{c} \Delta r^{2}}{c}\right]
$$

where $Z_{0} \approx 376.7 \Omega$ is the vacuum impedance; and $\Delta r$ is the thickness of the resistive wall. In particular $\mathcal{W}(0)=0$, and $\mathcal{W}(\zeta)$ decays faster than $\zeta^{-1 / 2}$ when $\zeta \rightarrow \infty$ since $\int_{0}^{\infty} \mathcal{W}(\zeta) d \zeta$ - the dc impedance - is finite. It is convenient to introduce a characteristic length of the resistive wall wakefield as

$$
s_{0} \equiv\left(\frac{2 b^{2}}{Z_{0} \sigma_{c}}\right)^{1 / 3}
$$

For the LCLS project at SLAC, the FWHM bunch length is less than ten times of $s_{0}$. Furthermore, the resistive-wall wakefield is stronger at short-range. Hence, it is important to look into short-range wakefield effects.

\section{Dipole Mode}

There has been study for the monopole mode and the corresponding short-range longitudinal effect [3]. Here, in

\footnotetext{
* The work of AWC and JW was supported by the US Department of Energy under contract DE-AC02-76SF00515. The work of JRD was supported by US Department of Energy under contract No. DE-AC0506OR23177.

$\dagger$ jhwu@SLAC.Stanford.EDU
}

\begin{tabular}{lc} 
Table 1: Parameters for the LCLS project. \\
\hline \hline bunch rms duration $\sigma_{B}(\mathrm{ps})$ & 0.077 \\
Pipe radius $\mathrm{b}(\mathrm{cm})$ & 0.3 \\
Pipe length $\mathcal{L}(\mathrm{m})$ & 150 \\
Conductivity $\sigma_{c}\left(10^{7} \Omega^{-1} \mathrm{~m}^{-1}\right)$ & 6 \\
$\kappa\left(\mathrm{m}^{-1}\right)$ & $1 / 18$ \\
Beam energy $(\mathrm{GeV})$ & 14.35 \\
Bunch charge $(\mathrm{nC})$ & 1 \\
\hline$\delta_{\text {skin }}\left(\omega=\sqrt{3} / \tau_{0}\right)(\mathrm{nm})$ & 21.8 \\
$s_{0}(\mu \mathrm{m})$ & 9.3 \\
$\tau_{0}(\mathrm{ps})$ & 0.031 \\
$\varepsilon\left(10^{9} \mathrm{~s}^{-1} \mathrm{~m}^{-2}\right)$ & -11.9 \\
\hline \hline
\end{tabular}

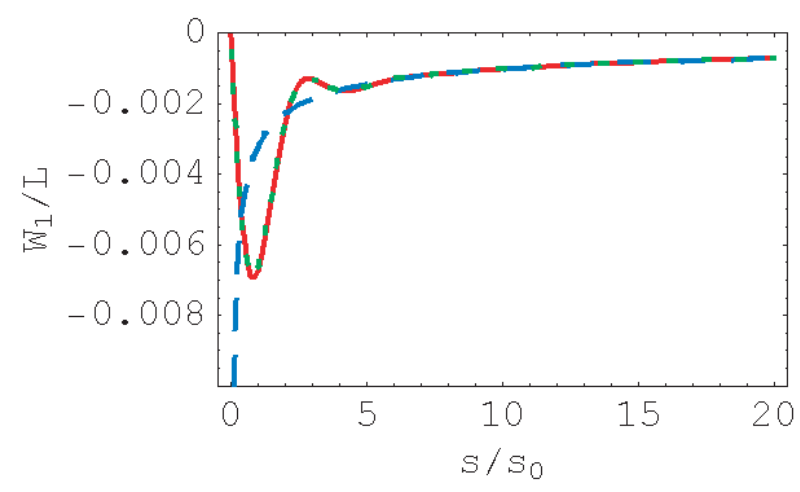

Figure 1: (Color) Plot of the wake function per unit length $W_{1}(s) / L$ as a function of $s / s_{0}$. The solid(red) curve stands for the full wake, the dashed(blue) curve for the long-range wake, and the dotted(green) curve for the numerical results with all three terms in the denominator of Eq. (4).

this paper, we study the dipole mode wakefield and the corresponding short-range transverse effect.

The wake functions are defined as that in Ref. [2]

$$
\int_{-L / 2}^{L / 2} d s F_{\|}=-e I_{m} W_{m}^{\prime}(z) r^{m} \cos (m \theta),
$$

where $m$ stands for the $m$-th mode with $(r, \theta)$ being the cylindrical transverse coordinates; $I_{m}$ is the $m$-th moment of the beam, $W_{m}$ is the wake due to the $m$-th mode, $e$ is the electron charge, and $F_{\|}=e E_{s}$ is the longitudinal force. For $m=1$, we solve the problem in frequency domain $\left[E_{s}(z)=\int_{-\infty}^{\infty}(d k / 2 \pi) e^{i k z} \tilde{E}_{s}\right]$ to get

$$
\tilde{E}_{s}=\frac{4 I_{1} r}{b^{3}\left(\frac{i k b}{2}-\frac{\lambda}{k}+\frac{i}{k b}\right)} \approx \frac{4 I_{1} r}{b^{3}\left(\frac{i k b}{2}-\frac{\lambda}{k}\right)}, \text { for } r<b
$$


where the $i /(k b)$ term has been dropped in the denominator. This is valid for the frequency regime

$$
\lambda \gg k^{2} b \gg \frac{1}{b} .
$$

Recall that, $\lambda=\sqrt{\sigma_{c} Z_{0}|\omega| /(2 c)}[i+\operatorname{sgn}(\omega)]$ with $\sigma_{c}$ being the conductivity. The parameter $\lambda^{-1}$ is related to the skin depth as a function of frequency $\omega=k c$ inside the metal:

$$
\delta_{\text {skin }}=\frac{1}{\operatorname{Im} \lambda}=\sqrt{\frac{2 c}{\sigma_{c} Z_{0}|\omega|}} .
$$

Hence, the assumption in Eq. (5) means that the pipe radius is much larger than the impedance wavelength, which is much larger than the skin depth. Since the characteristic length $s_{0}$ stands for the typical wavelength of the impedance we are interested, we show $\delta_{\text {skin }}, s_{0}$, and $b$ in Table 1. In Table 1, the skin depth $\delta_{\text {skin }}$ is evaluated at $|\omega|=\sqrt{3} c / s_{0}$ as inspired by the full wake (MKS units)

$$
\frac{W_{1}(\tau)}{L}=\left\{\begin{array}{cc}
-\frac{1}{\pi b^{3}} \sqrt{\frac{1}{\pi \varepsilon_{0} \sigma_{c}}} \frac{1}{\sqrt{\tau}} \quad \text { for } \tau \gg \tau_{0} \\
\frac{8 \tau_{0} c}{\pi \varepsilon_{0} b^{4}}\left[\frac{1}{12} e^{-\tau / \tau_{0}} \cos \left(\sqrt{3} \tau / \tau_{0}\right)\right. \\
-\frac{1}{4 \sqrt{3}} e^{-\tau / \tau_{0}} \sin \left(\sqrt{3} \tau / \tau_{0}\right) \\
\left.-\frac{\sqrt{2}}{\pi} \int_{0}^{\infty} d x \frac{e^{-x^{2} \tau / \tau_{0}}}{x^{6}+8}\right]
\end{array}\right.
$$

where $\tau=s / c, \tau_{0}=s_{0} / c$. We state the validity criteria in Eq. (5) for obtaining the short-range wake as in Eq. (7). For comparison, we can also numerically obtain the short-range wakefield with all the three terms included in the denominator of Eq. (4). Recall that we throw away the third term $i /(k b)$ in the denominator of Eq. (4) to obtain the analytical expression in Eq. (7). The numerical result is shown as the dotted (green) curve in Fig. 1, where the short-range analytical expression in Eq. (7) is shown as the solid (red) curve, and the long-range expression in Eq. (7) is shown as the dashed (blue) curve. There is essentially no difference with or without the third term $i /(k b)$ for our example. On the other hand, the long range expression is accurate only when $s>3 s_{0}$.

\section{Equation of Motion}

In a continuum approximation, the transverse motion $x(\zeta, s)$ of a beam in a misaligned beamline under the influence of focusing and wakefield can be modelled by $[4,5]$

$$
\begin{gathered}
\frac{\partial^{2} x(\zeta, s)}{\partial s^{2}}+\kappa^{2}\left[x(\zeta, s)-d_{f}(s)\right]=-\frac{N e^{2}}{\sqrt{2 \pi} \sigma_{t} \gamma m v^{2}} \frac{v}{c} \\
\times \int_{0}^{\zeta} d \zeta^{\prime} \frac{W_{1}\left(\zeta-\zeta^{\prime}\right)}{L} F\left(\zeta^{\prime}\right)\left[x\left(\zeta^{\prime}, s\right)-d_{c}(s)\right],
\end{gathered}
$$

where $\kappa$ is the wave number representing the betatron focusing strength; $F(\zeta)=I(\zeta) / \bar{I}$, the current form factor, is the instantaneous current $I(\zeta)$ divided by the average current $\bar{I} \equiv Q /\left(\sqrt{2 \pi} \sigma_{t}\right)$ with $Q=N e$ the bunch charge and $\sigma_{t}$ the rms bunch duration; $d_{f}(s)$ and $d_{c}(s)$ are the lateral displacements of the focusing elements and of the beam transport components where the wakefield is generated, respectively. The right hand side of Eq. (8) represents the effects due to the wakefield.

We restrict ourselves for the case of $d_{f}(s)=d_{c}(s)=0$. Hence, the equation of motion (8) is simplified as

$$
\begin{gathered}
\frac{\partial^{2} x(\zeta, s)}{\partial s^{2}}+\kappa^{2} x(\zeta, s)=\varepsilon \int_{0}^{\zeta} d \zeta^{\prime} w\left(\zeta-\zeta^{\prime}\right) F\left(\zeta^{\prime}\right) x\left(\zeta^{\prime}, s\right), \\
\text { where } \varepsilon=-\frac{32 s_{0} c}{\gamma b^{4}} \frac{\bar{I}}{I_{\text {Alfvèn }}}, \quad \text { and } \\
w(\zeta)=\frac{e^{-\zeta / \tau_{0}}}{12} \cos \left(\frac{\sqrt{3} \zeta}{\tau_{0}}\right)-\frac{e^{-\zeta / \tau_{0}}}{4 \sqrt{3}} \sin \left(\frac{\sqrt{3} \zeta}{\tau_{0}}\right) \\
-\frac{\sqrt{2}}{\pi} \int_{0}^{\infty} d x \frac{e^{-x^{2} \zeta / \tau_{0}}}{x^{6}+8} .
\end{gathered}
$$

\section{Series Solution for Arbitrary Bunch Shape}

With the full wake, we can take the series solution approach [6] to study the transverse wake effect for electron bunch with arbitrary longitudinal shape. In this paper, we will not discuss the misalignment effect, hence we set $d_{f}(s)=d_{c}(s)=0$ to get [6]

$$
\begin{aligned}
x(s, \zeta) & =\sum_{n=0}^{\infty} \varepsilon^{n}\left[x_{0} h_{n}(\zeta) j_{n}(\kappa, s)+x_{0}^{\prime} g_{n}(\zeta) i_{n}(\kappa, s)\right] \\
\equiv & x_{0} \mathcal{A}(s, \zeta ; \varepsilon)+x_{0}^{\prime} \mathcal{B}(s, \zeta ; \varepsilon), \\
x^{\prime}(s, \zeta) & =x_{0}^{\prime} \sum_{n=0}^{\infty} \varepsilon^{n} g_{n}(\zeta) j_{n}(\kappa, s) \\
& +x_{0}\left\{-\kappa^{2} i_{0}(\kappa, s) h_{0}(\zeta)\right. \\
& \left.+\sum_{n=1}^{\infty} \varepsilon^{n} h_{n}(\zeta)\left[i_{n-1}(\kappa, s)-\kappa^{2} i_{n}(\kappa, s)\right]\right\} \\
& \equiv x_{0} \mathcal{C}(s, \zeta ; \varepsilon)+x_{0}^{\prime} \mathcal{D}(s, \zeta ; \varepsilon),
\end{aligned}
$$

where the transfer functions $\mathcal{A}, \mathcal{B}, \mathcal{C}$, and $\mathcal{D}$ are introduced.

$$
\left(\begin{array}{c}
x(s, \zeta) \\
x^{\prime}(s, \zeta)
\end{array}\right)=\left(\begin{array}{cc}
\mathcal{A}(s, \zeta ; \varepsilon) & \mathcal{B}(s, \zeta ; \varepsilon) \\
\mathcal{C}(s, \zeta ; \varepsilon) & \mathcal{D}(s, \zeta ; \varepsilon)
\end{array}\right)\left(\begin{array}{c}
x_{0} \\
x_{0}^{\prime}
\end{array}\right)
$$

The functions $g_{n}(\zeta)$ and $h_{n}(\zeta)$ are defined recursively

$$
\left\{\begin{array}{l}
g_{n+1}(\zeta) \\
h_{n+1}(\zeta)
\end{array}\right\}=\int_{0}^{\zeta}\left\{\begin{array}{l}
g_{n}\left(\zeta_{1}\right) \\
h_{n}\left(\zeta_{1}\right)
\end{array}\right\} w\left(\zeta-\zeta_{1}\right) F\left(\zeta_{1}\right) d \zeta_{1},
$$

where

$$
\left\{\begin{array}{l}
x_{0}^{\prime} g_{0}(\zeta)=x_{0}^{\prime}(\zeta)=\left.\frac{\partial}{\partial s} x(s, \zeta)\right|_{s=0} \\
x_{0} h_{0}(\zeta)=x_{0}(\zeta)=x(s=0, \zeta)
\end{array}\right.
$$

and $x_{0}(\zeta)$ and $x_{0}^{\prime}(\zeta)$ are the lateral displacement and angular divergence, respectively, of the beam at the entrance of the accelerator. The normalizing constants $x_{0}$ and $x_{0}^{\prime}$ 

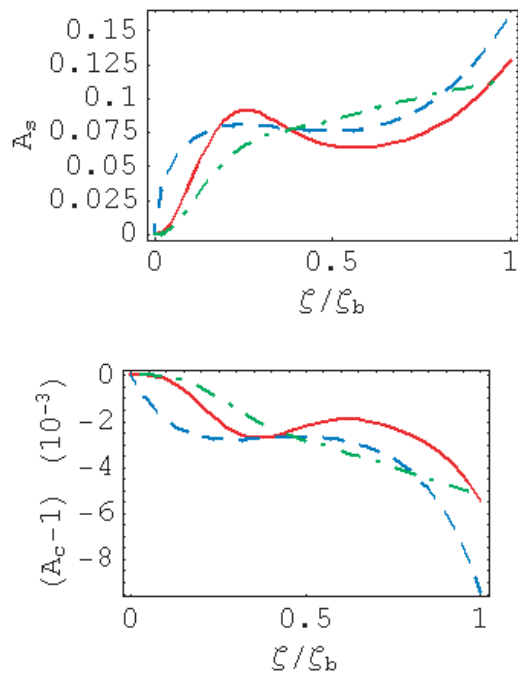
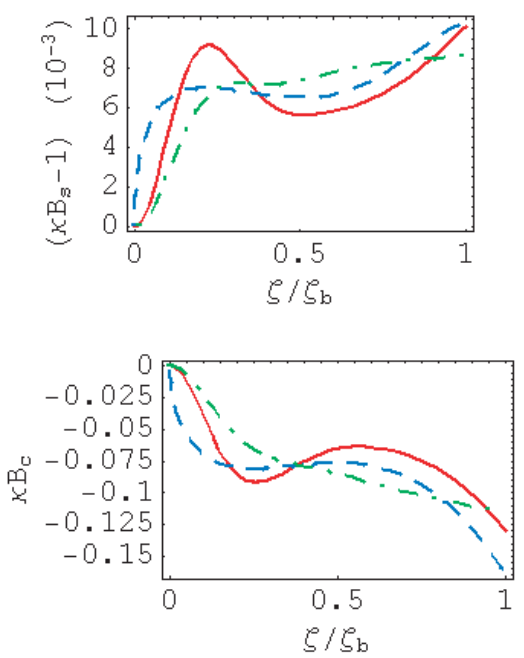
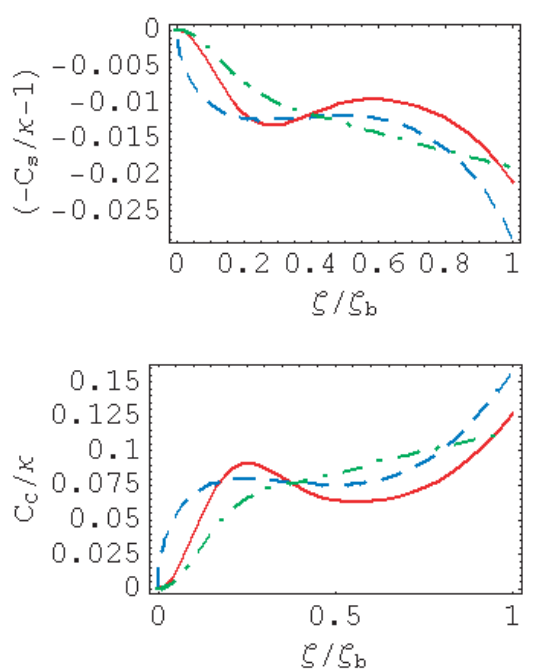

Figure 2: (Color) Plot of $\mathcal{A}_{s}, \kappa \mathcal{B}_{s}-1,-\mathcal{C}_{s} / \kappa-1, \mathcal{A}_{c}-1, \kappa \mathcal{B}_{c}$, and $\mathcal{C}_{c} / \kappa$ along the bunch with double-horn distribution for LCLS. The solid (red) curves are obtained with the full wake as in Eq. (11) or the second expression in Eq. (7). The dashed (blue) curves are obtained with the long-range wake as the first expression in Eq. (7). For comparison, the short-range wake and uniform distribution case is shown as the dot-dashed (green) curve.

are introduced to make the functions $h_{0}(\zeta)$ and $g_{0}(\zeta)$ dimensionless. In particular if the injection offsets are timeindependent, which is what we assume for the remainder of the paper, we have $g_{n}(\zeta)=h_{n}(\zeta)$. Note that, since Eq. (8) assumes that the beam was turned on at $\zeta=0$, the functions $g_{n}(\zeta)$, and $h_{n}(\zeta)$ are defined only for $\zeta>0$. The functions $i_{n}(\kappa, s)$ and $j_{n}(\kappa, s)$ are defined in terms of Bessel functions of order integer plus one half,

$$
\left\{\begin{array}{l}
i_{n}(\kappa, s)=\frac{1}{n !}\left(\frac{s}{2 \kappa}\right)^{n} \frac{1}{\kappa} \sqrt{\frac{\pi \kappa s}{2}} J_{n+(1 / 2)}(\kappa s) \\
j_{n}(\kappa, s)=\frac{d}{d s} i_{n}(\kappa, s)=\frac{s}{2 n} i_{n-1}(\kappa, s)
\end{array} .\right.
$$

\section{Application to LCLS Project}

For LCLS, the longitudinal current distribution has a double-horn structure. The head and tail of the bunch has a higher peak current. We model it as

$$
F(\zeta)=6\left(\frac{\zeta}{\zeta_{b}}-\frac{1}{2}\right)^{2}+\frac{1}{2}
$$

for $\zeta \in\left[0, \zeta_{b}\right]$, and $F(\zeta)=0$ otherwise. Given the doublehorn current distribution in Eq. (18) and the full wake in Eq. (11), we numerically calculate the general series solutions in Eqs. (12) and (13). With strong focusing the transfer functions are convenient to be written as

$$
\begin{aligned}
& \mathcal{A}(s, \zeta ; \varepsilon)=\mathcal{A}_{s}(s, \zeta ; \varepsilon) \sin (\kappa s)+\mathcal{A}_{c}(s, \zeta ; \varepsilon) \cos (\kappa s), \\
& \mathcal{B}(s, \zeta ; \varepsilon)=\mathcal{B}_{s}(s, \zeta ; \varepsilon) \sin (\kappa s)+\mathcal{B}_{c}(s, \zeta ; \varepsilon) \cos (\kappa s), \\
& \mathcal{C}(s, \zeta ; \varepsilon)=\mathcal{C}_{s}(s, \zeta ; \varepsilon) \sin (\kappa s)+\mathcal{C}_{c}(s, \zeta ; \varepsilon) \cos (\kappa s),(19) \\
& \mathcal{D}(s, \zeta ; \varepsilon)=\mathcal{D}_{s}(s, \zeta ; \varepsilon) \sin (\kappa s)+\mathcal{D}_{c}(s, \zeta ; \varepsilon) \cos (\kappa s) .
\end{aligned}
$$

These transfer functions are not independent but are related by $\mathcal{C}(s, \zeta ; \varepsilon)=\partial \mathcal{A}(s, \zeta ; \varepsilon) / \partial s$ and $\mathcal{D}(s, \zeta ; \varepsilon)=$ $\partial \mathcal{B}(s, \zeta ; \varepsilon) / \partial s$. Additionally, if the injection offsets are time-independent, we have $h_{n}(\zeta)=g_{n}(\zeta)$ and therefore $\mathcal{A}(s, \zeta ; \varepsilon)=\mathcal{D}(s, \zeta ; \varepsilon)$. In Fig. 2, we plot $\mathcal{A}_{s}, \kappa \mathcal{B}_{s}-1$, $-\mathcal{C}_{s} / \kappa-1, \mathcal{A}_{c}-1, \kappa \mathcal{B}_{c}$, and $\mathcal{C}_{c} / \kappa$ for the LCLS project with parameters in Table 1. The results with the full wake as in Eq. (11) or the second expression in Eq. (7) are shown in Fig. 2 as the solid (red) curves. To compare the results using the full wake with the results using the long-range wake, we also shown the results with the long range wake [ the first expression in Eq. (7) ] as the dashed (blue) curve in Fig. 2. To compare the results using the double-horn distribution with the results using a simple uniform distribution, we also shown the results with an uniform distribution and the full wake [ the second expression in Eq. (7) ] as the dot-dashed (green) curve in Fig. 2.

In this paper, we study the transverse effect from the full wake due to resistive wall, which is given in Eq. (7) together with the long-range wake. We work on a realistic double-horn current distribution as Eq. (18) which is found in LCLS project. The results are shown in Fig. 2.

\section{REFERENCES}

[1] P.L. Morton, V.K. Neil, and A.M. Sessler, J. Appl. Phys. 37, 3875 (1966).

[2] A.W. Chao, Physics of Collective Beam Instabilities in High Energy Accelerators, (Wiley-Interscience Publication, John Wiley \& Sons, Inc., New York, New York, 1993).

[3] K.L.F. Bane and M. Sands, SLAC Report No. SLAC-PUB7074, 1995.

[4] A.W. Chao, B. Richter, and C.-Y. Yao, Nucl. Instrum. Methods 178, 1(1980).

[5] Y.Y. Lau, Phys. Rev. Lett. 63, 1141(1989).

[6] J.R. Delayen, Phys. Rev. ST Accel. Beams 6, 084402 (2003). 